\title{
Aesthetic Preferences in Mathematics: a Case Study
}

\section{Abstract}

Although mathematicians often use it, mathematical beauty is a philosophically challenging concept. How can abstract objects be evaluated as beautiful? Is this related to the way we visualise them?

Using a case study from graph theory (the highly symmetric Petersen graph), this paper tries to analyse aesthetic preferences in mathematical practice and to distinguish genuine aesthetic from epistemic or practical judgements.

It argues that, in making aesthetic judgements, mathematicians may be responding to a combination of perceptual properties of visual representations and mathematical properties of abstract structures; the latter seem to carry greater weight. Mathematical beauty thus primarily involves mathematicians' sensitivity to aesthetics of the abstract.

\section{Introduction}

What is mathematical beauty? How could beauty be found in such an abstract subject as mathematics? Not attempting to solve this problem, I will attempt to answer a pair of specific questions which have some bearing on it. These questions arise from mathematicians' judgements about both the abstract objects they investigate and the visual artefacts they use to represent those objects. These seem to be aesthetic judgements, 
often using the word "beautiful", about the objects and their representations. Could such judgements, taken literally, be correct? Specifically:

(i) Can an abstract mathematical object be literally beautiful?

(ii) Can one of its visual representations be more beautiful than others?

The last question needs to be refined. A visual representation can be appreciated without regard for what it is intended to represent, and as such could be beautiful in a way that a non-mathematician could appreciate. But that is not what is meant. A better way to put the question is this:

(ii) Can one visual representation of a mathematical object represent its object in a more beautiful way than another visual representation represents the same object? And what does this mean for a mathematician?

Mathematicians' talk suggests a positive answer to both questions. But the possibility of loose or metaphorical uses of aesthetic expressions entails that we should be cautious about taking such talk at face value. In what follows I will first very briefly set out some of the positions that philosophers working in aesthetics have taken related to these questions. I will then introduce a particular mathematical example, an abstract object and representations of it, and mathematicians' apparently aesthetic judgements about them. Finally, I will discuss these examples with a view to answering my questions.

\section{The ongoing discussion in aesthetics}

There is an on-going discussion about whether there is such a thing as mathematical beauty, since there seem to be two options to consider, namely that beauty, apart from sharing a propensity to give pleasure of a certain disinterested kind (i.e. noninstrumental pleasure), is: 
1. only perceptual, i.e. dependent only on properties, which an object can be perceived to have; all the other talk about "beauty" is metaphorical or loose or

2. not-only perceptual, but also intellectual (and possibly of many kinds).

Clearly it is only in case 2 that there could be mathematical beauty, strictly and literally speaking. Jerrold Levinson [2011] among others claims that there is an irreducible variety of beauty, supporting claim 2. Rafael De Clercq [2015] in his excellent survey briefly criticises the postulation of different kinds of beauty and calls attention to the problem of expert-related aesthetic judgements as a way to define beauty. Nick Zangwill [2003, 2001, 1999] supports claim 1 and in considering mathematical proofs, denies that they are literally elegant. John Barker [2009] responds to Zangwill's arguments and puts forward the idea that elegance of mathematical proofs is the same elegance as found in other objects. I will consider Zangwill's points as they apply to certain mathematical entities which are not proofs. Most works discussing mathematical beauty, including Barker's, concentrate on proofs. Zangwill builds his argument on the basis that a proof is an epistemically functional construction and all aesthetic-like appreciations of elegant proofs are strictly speaking epistemic rather than aesthetic. I will examine aesthetic judgements of experts in a particular area. I will point out that a closer look at mathematical practice shows that mathematical beauty is not limited to proofs only: there are other entities appreciated as beautiful. In this paper I will consider mathematical graphs rather than proofs, where these are the objects of graph theory, not the diagrams found in calculus textbooks and elsewhere.

I start the paper with some basic assumptions about beauty. I then explain the distinction between an abstract mathematical object and its particular visualisations. I distinguish beauty of the object and beauty of its visual representations. This is not a metaphysical but a methodological distinction, which will later help me to trace the contributors to mathematical beauty. Looking at particular cases, I identify loose and 
correct uses of the word "beautiful" in mathematicians' judgements and also the factors of beauty to which mathematicians respond and whether these factors are perceptual or not. I take some time to develop my case study and towards the end I return to the philosophical discussion and respond to some objections.

\section{My assumptions}

Let me begin with some minimal understanding of mathematical beauty and then try to develop this understanding by studying particular examples. As mentioned Zangwill's view is disputed by Barker and others, and I will take it as a premise in this paper that abstract mathematical entities can be beautiful, strictly and literally speaking.

To judge something mathematical to be beautiful is to admit that this thing has the power to give an aesthetic pleasure and which is valued to some positive degree. By saying "This is a beautiful proof!" the mathematician implies that the proof has a positive emotional effect on her in comparison to other proofs she knows. This does not imply that beauty is a particular property of a mathematical entity independent of a mathematician's cognition, like the curvature of a surface or the number of roots of an equation. Beauty seems to be more like a power, causing pleasure to mathematicians while they are intellectually engaged with the mathematical entity. In aesthetic terms, beauty is a response-dependent property.

I do not want to go into discussion about objectivity of beauty, but it is important to distinguish the use of "beautiful" to make an aesthetic judgement from various loose uses of the word, for example, when something is called "beautiful" because it appeals to (or satisfies) a practical interest or helps to reach a goal. "I will give you now a beautiful example", may be said when the speaker likes the example only because it is typical and easy to understand, i.e. satisfies somebody's practical interests. 
An aesthetic judgement is not merely an expression of feeling about the mathematical entity. In saying of something that it is beautiful, the speaker is not saying that it delights her or that she likes it very much. For on the question of what (currently) delights her she is the authority; but on the question of the aesthetic quality of the entity, she is open to education and correction by people of greater expertise and more developed sensibility of the relevant kind.

In this paper I constrain the discussion to aesthetic judgements made by specialists about their subject matter. While there is rarely perfect agreement about what is beautiful and what is not, there is a large measure of agreement among specialists when making comparisons. ${ }^{1}$

Another assumption I make is that (mathematical) beauty is a matter of degree. We can judge one entity to be more beautiful than another, and some can even appear ugly to us. In practice aesthetic judgements often arise in the context of comparing things. A mathematics lecturer will not miss a chance to mention to the students if somebody has found a more beautiful proof of a theorem than the one in their textbook; and she will be always delighted by a student's more beautiful solution to a problem. Analogously, as I will show later, one representation of a mathematical object may be judged more beautiful than another.

\section{Mathematical objects and their cognitive represent- ations}

Mathematical matter is abstract, but in practice mathematicians use representations of mathematical entities such as drawings of graphs, diagrams, written formulas, tables

\footnotetext{
${ }^{1}$ For the proponents of response-dependent view see Goldman [1995] and Levinson [2011]; for the criticism see Budd [2008] and De Clercq [2015].
} 
and the like. These representations, along with definitions, help to fix the abstract content and help understanding and communication; they also facilitate various mathematical actions (such as matrix multiplication). For a simple example, a linear function is a mathematical object, but the formula printed here: " $\mathrm{y}=\mathrm{x}+5$ " or a visualised graph of this function are cognitive representations of this function. The distinction between an abstract mathematical object and its cognitive (visual) representation will be useful in analysing aesthetic judgements in mathematics.

\section{Perceptual vs. intellectual beauty}

I will distinguish perceptual and intellectual (or mathematical) beauty, in respect to mathematical visualisations and mathematical objects. Kant already said that "beautiful representations of objects are to be distinguished from beautiful objects", and I will come back to his view later in the discussion. ${ }^{2}$ I am not saying that they are two different kinds of beauty; I simply want to point out that mathematicians when judging a mathematical entity as beautiful, may be responding to abstract properties of mathematical objects or to visible properties of the representation. Perceptual beauty can be found in visually pleasing representations of mathematical entities, such as visualisations of geometric constructions, graphs and arrays of formulas. Looking at a visual representation we can appreciate its perceptual beauty.

In that case it is not the mathematical content of the representation that causes us pleasure. It is possible that somebody without any mathematical background and who does not know or understand the mathematical content of the representation can appreciate the overall visual effect of the mixture of visible properties, such as symmetry, smoothness of curves and the combination of colours.

\footnotetext{
${ }^{2}$ I borrow this quotation from Wenzel [2001], p. 426 and I am grateful for the referee for recommending it to me.
} 
To illustrate the case of intellectual beauty, I will use a puzzle "tangram", originated in China. ${ }^{3}$ It consists of seven flat pieces, which may not overlap and aim to form different shapes. The conventional default shape is a square as in Figure 1 a). Any particular shape is not what makes it special but that the fact that so many different shapes can be made is truly astonishing. Over 6500 different tangram problems can be found in the 19th century books alone, and the current number is increasing. ${ }^{4} \mathrm{New}$ mathematical facts have been revealing about tangram. For example, there are only 13 convex configurations (such that a line segment drawn between any two points on the configuration's edge always pass through the configuration's interior, i.e., configurations with no recesses in the outline) as shown in Figure $1 \mathrm{~b}) .{ }^{5}$ So it is not the shapes themselves but their numerosity and diversity that is attractive. That could be classified as intellectually pleasing.
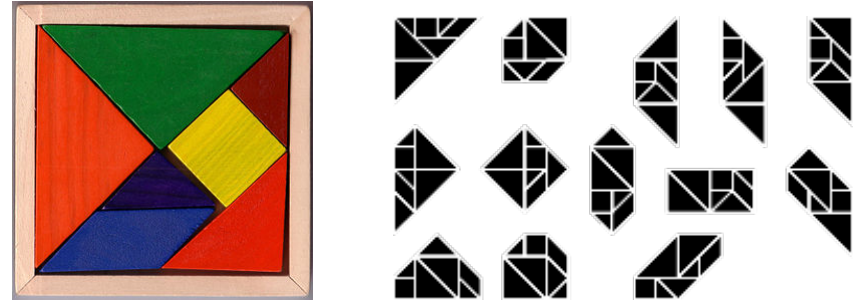

Figure 1: a) Tangram standard view; b) Tangram 13 possible convex shapes.

A clash between visualisation and geometrical combinatorics behind it can create paradoxes. Some visualisations surprise us: they seem to be broken, but in fact they contain the same seven geometric shapes. In Figure 2 a) depicting the Two monks paradox the first monk is missing a foot; as do the second and third cups in the right figure b), the Magic Dice Cup tangram paradox. ${ }^{6}$ As soon as we understand the paradox geometrically we are able to admire tangram's mathematical beauty.

\footnotetext{
${ }^{3}$ I am grateful to the referee for suggesting this example.

${ }^{4}$ Slocum [2001], p. 37.

${ }^{5}$ Proved in Traing Wang and Hsiung [1976].

${ }^{6}$ Loyd [1968], p. 25.
} 

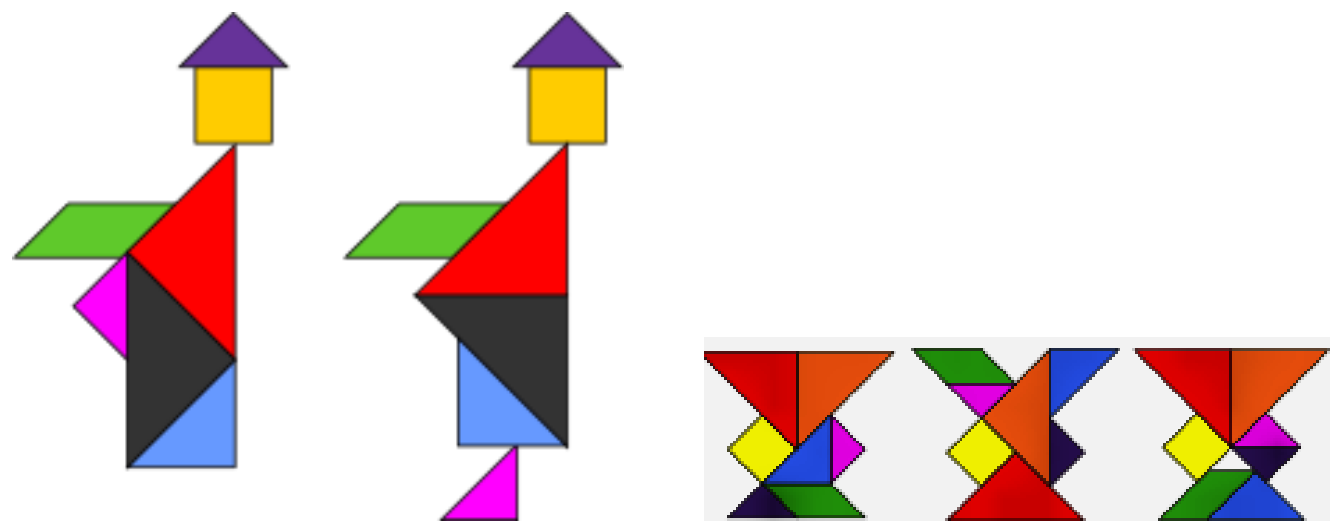

Figure 2: The two monks paradox; b) The Magic Dice Cup tangram paradox.

There is another observation. It looks like this judgement comes to us at once without distinguishing whether we appreciate the simplicity, clearness or arrangement of the diagram, or the clever management of perceptual elements of the diagram to serve the justification. Although visual representations are involved and understanding of the mathematics does rely on them, it is clearly non-perceptual beauty that initiates aesthetic judgements. This last observation brings us to the questions: How do perceptual features of visual representations of something mathematical interact with intellectual beauty of what is represented in the visualisation? Does the perceptual combined with the intellectual contribute to mathematical beauty? The strategy I will use to approach the questions above will be to (i) consider a particular property, which has traditionally been associated with beauty both in perceptual and intellectual sense, namely, symmetry and then (ii) examine how visually perceived symmetries and symmetries known to exist but not perceived can contribute to beauty; (iii) for this purpose I will focus on a particular mathematical example. 


\section{Symmetry as we see it and symmetry in mathematics}

In this section I will point out that not all abstract symmetries can be easily perceived in visual representations. In this case it would be useful to understand how the perceived and how simply calculated symmetries contribute to beauty. In fact only some symmetries of an object, usually reflection, rotation and translation, but not all of them, can be perceived in its visual representation. Examples for limits of visible symmetry will follow. For rotations and reflections of a square and a repeated pattern in tiles, are examples of symmetries we easily grasp. The distinction between symmetries visualised in representations and algebraically determined symmetries is sometimes designated as "geometrical and combinatorial symmetries"; I will use these expressions in the same way.

In mathematics "symmetry" has a precise connotation. ${ }^{7}$ A symmetry of a mathematical object is a transformation that leaves the object essentially unchanged: a structure preserving bijection of the object onto itself. From a mathematical perspective, there are many more symmetries than we can be perceptually sensitive to, partly because our natural grasp of symmetry is often solely geometrical.

However, symmetry has a broader scope than solely geometry. Consider the value preserving permutation of the product of two differences $(a-b)(c-d)$. These are algebraic symmetries.

\section{Symmetry in graph theory}

In discrete mathematics, a graph is an ordered pair $G=(V, E)$ where $V$ is a non-empty set of vertices of $G$ and $E$, the set of edges of $G$, is a subset of the 2-subsets of $V$. That is, the edges are (possibly ordered) pairs of the elements of $V$. For instance

\footnotetext{
${ }^{7}$ Weyl [1952] gives a nice exposition about symmetry and mathematics.
} 
the following set is a graph called $\mathrm{K}_{1,3}: K_{1,3}=\{V, E\}=\{\{u, v, w, x\},\{a, b, c\}\}=$ $\{\{u, v, w, x\},\{\{u, x\},\{v, x\},\{w, x\}\}\}$. In practice it is often useful to give a graph a pictorial representation: that is representing vertices as dots and edges as lines joining the relevant dots. Such pictorial representations can be realised in an aesthetically more or less attractive way, and this is were the question of aesthetics about representations appears.

In graph theory a symmetry is a vertex permutation that preserves graph structure, namely adjacency, non-adjacency of vertices and the number of edges between them.

Let me explicitly articulate the difference between (1) a geometrical symmetry and (2) a symmetry in graph theory.

(1) For any geometrical object $G$ (taken as a set of points in a Euclidean space of the relevant dimension) a symmetry $s$ of $G$ is a distancepreserving bijection from $G$ onto $G$. $s$ is distance-preserving iff for any $x, y$ in $G, d(s(x), s(y))=d(x, y)$;

(2) For any graph $G$ a symmetry $s$ of $G$ is an adjacency-preserving bijection of $V(G)$ onto $V(G)$, where $V(G)$ is the set of vertices of $G$. $s$ is adjacency-preserving iff for any $u, v$ in $V(G), u, v$ is an edge of $G$ iff $s(u), s(v)$ is an edge of $G$.

The symmetries that we notice in a graph drawing are those geometrical symmetries which can easily be discerned by visualising rigid motions of the whole geometrical object. Motions of this kind which take vertices to vertices will clearly preserve adjacency. So the vertex-to-vertex geometrical symmetries which we notice in a drawing of a graph reveal to us some of the graph symmetries. 


\section{The Petersen graph}

Graphs are convenient objects for observing symmetries: they are algebraic objects, and algebra provides us with sufficient information about combinatorial symmetries; at the same time, graphs have many visual representations, which are useful for studying perceptual aspects of symmetry including aesthetical aspects. Let me now introduce a graph which by many graph-theorists is considered to be "one of the most beautiful graphs in graph theory", the Petersen graph. Although the graph first appeared in Kempe [1886], it was named after Petersen (Petersen [1898]). In fact there are many drawings of this graph: symmetric and asymmetric. In Petersen's paper the drawing of this graph did not reflect its symmetries as in Figure 3a.
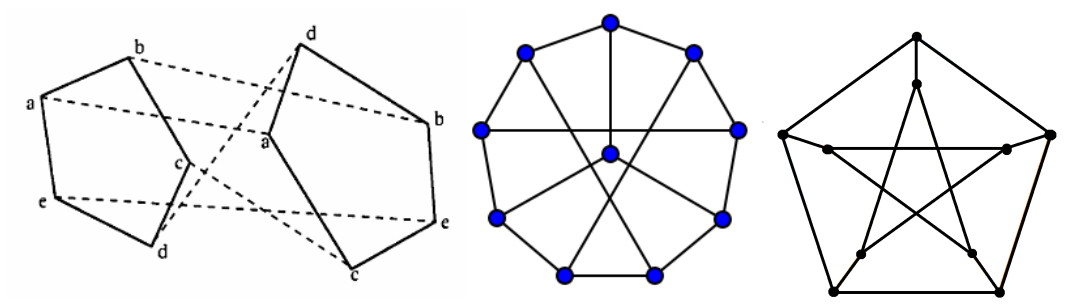

Figure 3: Three drawings of the Petersen graph: a) from Petersen's paper; b) from Kempe's paper; c) the traditional drawing.

In Kempe's paper the drawing appears with symmetry of order 3 as in Figure 3b, meaning the three rotations and three reflections. The most traditional and most preferred by mathematicians drawing of the Petersen graph as in Figure 3c shows 10 symmetries: five rotations and five reflections. In fact, it can be shown that a drawing of the Petersen graph can have at most ten symmetries, and is maximally symmetric. ${ }^{8}$

${ }^{8}$ This claim can be foud in Hong and Eades [2005]. Here is a sketch of the proof:

1. A group of symmetries of a graph drawing with more than 4 elements is dihedral (its automorphisms are reflections and rotations), and fixes at most one vertex.

2. Thus if a group of symmetries of the Petersen graph (which has 10 vertices and therefore the number of its automorphisms is 20) has a size $n$, then by Lagrange theorem (the order of every subgroup $H$ of the group $G$ divides the order of $G$ ), either $n$ divides 20 or $n$ divides 18 (in cases if there is a fix vertex). 
A striking fact is that the Petersen graph has 120 symmetries! Various drawings of the Petersen graph enable us to perceive some of its symmetries, but no drawing can show all the graph symmetries at once. We can visualise some rotations and reflections, but not all their compositions. So there is more mathematically detectable symmetry, or "invisible" or "hidden" beauty, as mathematicians like to put it. Having a variety of drawings of the Petersen graph, symmetric and asymmetric, is useful for different tasks, but interestingly one of them is considered to be "more beautiful" than the

others. Keeping in mind these two considerations: "hidden beauty" and "the most beautiful drawing", one about abstract and another about an artefact, I will try to explain this situation and explore the aesthetic impact of symmetry, starting with the impact of symmetries in an abstract mathematical sense, including those which are not visualised, as factors in beauty. Towards the end of paper I will consider how the visible symmetries can contribute to mathematical beauty.

\section{Is the Petersen graph strictly and literally beautiful?}

Having discussed aesthetics of visual representations of mathematical entities I now move to the aesthetics of the abstract mathematical entities themselves. In this case the entity is the Petersen graph, independently of a particular representation, i.e. a collection of vertices connected by edges, but not the drawings. From this perspective, how could anything so abstract as that be beautiful?

My general answer to this question is that a combination of rare and eminent math-

3. The Petersen graph automorphism group is S5, which does not contain elements of order 9 and 10 and therefore has no cyclic automorphism of size 9 and 10.

4. Thus the dihedral group of size 10 (cycle of size 5 plus a reflection) is the largest possible. 
ematical properties of the abstract object shapes the intellectual beauty to which mathematicians respond emotionally. If one asks graph theorists who work with the Petersen graph why it is beautiful, they naturally give an extended list of reasons.

Compared to many important graphs, e.g. the Hoffman-Singleton graph (see Figure 4 right-hand side), the Petersen graph has relatively few vertices and edges. Yet mathematicians have discovered that it has a multitude of outstanding qualities, which one would not expect from initial acquaintance. It is first of all this combination of properties that motivates the strongly positive aesthetic response of mathematicians. They are responding to what they have discovered of the deep nature of the Petersen graph, as opposed to its surface appearance in visual representations. In order to give the reader a taste of what mathematicians are responding to, a brief description of several relevant properties will be given in the next few paragraphs (not all can be defined here). ${ }^{9}$

\section{Rich in symmetry}

The Petersen graph is highly symmetric. There are 120 symmetries - permutations of vertices and edges preserving adjacency/non-adjacency. Graph theory also interprets them in terms of various regularities. The Petersen graph is vertex-transitive, meaning there are symmetries taking any one vertex to any other (every vertex has the same adjacency/non-adjacency configuration). Consequently, it is strongly regular: (a) any two adjacent vertices have the same number $k$ of common neighbours as any other two adjacent vertices and (b) any two non-adjacent vertices have the same number $j$ of common neighbours as any other two non-adjacent vertices. It is also edge-transitive, which means, analogously to vertex-transitivity, that there are symmetries taking any

\footnotetext{
${ }^{9}$ The choice of properties is suggested in Goucher [2013].
} 
one edge to any other. In fact, it is 3-arc-transitive: every directed three-edge path in the Petersen graph can be transformed into every other such path by an automorphism. The Petersen graph is also a cubic graph. A cubic graph is a graph in which all vertices have degree three, as it is in a graph representing the edges and vertices of a cube. In combination with symmetry cubic graphs form a list called Foster census of only thirteen cubic symmetric graphs with up to 30 vertices. Only ten of them are also distance transitive. Remarkably the Petersen graph is one of only ten cubic distance-regular graphs! $!^{10}$

This high degree of symmetry in such a small graph is impressive, and regardless of our visual perception of it, symmetry makes a mathematical object more beautiful. Why? Firstly, because symmetry helps us to grasp mathematical structure and its higher generality. Indeed, on the basis of these different types of symmetries one can form families of graphs and compare them by relating properties. Also some of these types form hierarchies: e.g. strong regularity implies regularity, distance transitivity implies arc transitivity, which implies vertex and edge-transitivity, and etc. Thus the classification of these symmetries provides an account for generality in studying graphs. Secondly, symmetry may lead us to other significant properties and connections. Let me now indicate this with some examples.

\section{One of four possible Moore graphs}

The Petersen graph is one of only four possible Moore graphs. This is an exceptional property, which is directly related to symmetry, namely strong regularity of the graph, and depends on two parameters: diameter and girth. A graph diameter is the greatest of the shortest distances between two vertices and since in the Petersen graph any two

\footnotetext{
${ }^{10}$ In a distance-regular graph, for any two vertices $u$ and $v$ at distance $k$, the number of neighbours of $v$ that are at distance $k$ from $u$ is the same.
} 
vertices can be connected by two edges the graph diameter is 2 . The girth of a graph is the length of a shortest cycle contained in the graph (shortest closed path without repeating vertices except the starting one). In a n-regular graph every vertex is adjacent to n edges. For diameter 2 and girth 5 the only possible strongly regular graphs are:

1. The 5-vertex 2-regular pentagon;

2. The 10-vertex 3-regular Petersen graph;

3. The 50-vertex 7-regular Hoffman-Singleton graph;

4. The 3250-vertex 57-regular graph (open problem).

The first three are depicted in Figure 4 below, and the fourth is not proven to exist yet.
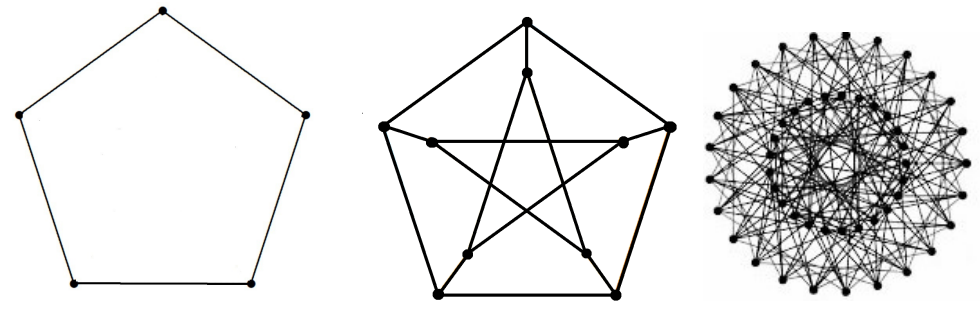

Figure 4: Three known Moore graphs: pentagon, the Petersen and the HoffmanSingleton graphs.

To be a Moore graph is therefore a rare property; it is intriguing and surprising. This is what may help to make these graphs seem beautiful to mathematicians. Moreover, the Petersen graph has a combination of virtues not shared by the other two Moore graphs known to exist, pentagon and the Hoffman-Singleton graph. The Petersen graph is not as trivial as pentagon and has more interesting structure. At the same time it is not as complicated as the Hoffman-Singleton graph: its configuration is very clear and can be grasped at a glance. It is very likely that our aesthetic responses are more favourable when we find a balance between simplicity and complexity. So even among the Moore graphs it may be the most attractive one. Interestingly, one can notice in 
Figure 4 that the Hoffman-Singleton graph contains copies of the Petersen graph (in fact exactly 525). ${ }^{11}$

\section{Not a Cayley graph (not a graph of a group)}

The Petersen graph is vertex-transitive. All Cayley graphs, which are graphs of generated groups, have this property by construction. Curiously not all vertex-transitive graphs are Cayley. It was shown in Biggs [1994] that the Petersen graph is the smallest connected vertex-transitive graph that is not Cayley. The right graph, K5, in Figure 6, is a Cayley graph and the left one, the Petersen, is not. This is quite a surprising fact and it is due to the high symmetry of the Petersen graph. Indeed there are many vertex-transitive graphs, and many of them are Cayley graphs; we know how to construct those. Constructing vertex-transitive non-Cayley graphs, however, is a challenging ongoing project. Interestingly the Petersen graph falls into this special category.

\section{Non-planar}

A graph may have planar and non-planar drawings in a Euclidean plane. If a graph has any planar drawing, it is a planar graph as in Figure 5. Therefore a planar drawing proves the graph's planarity; otherwise we have to prove that such a drawing is impossible.

There are a few proofs of non-planarity. Some of them are based on the existence of particular configurations in the graph. The existence of such configurations is visible with the aid of symmetry of the Petersen graph. A graph is non-planar if and only if it

\footnotetext{
${ }^{11}$ See Klin and Zivav [2014], p.121.
} 

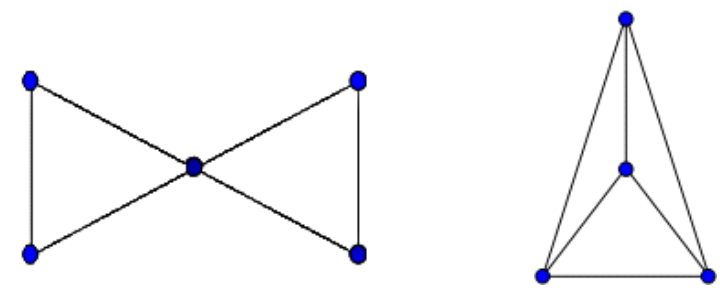

Figure 5: Examples of planar graphs.

contains graphs K5 or K3,3 as its minors (Klaus [1937]). A minor of a given graph is another graph formed by deleting vertices, edges and/or contracting edges. When an edge is contracted, its two vertices are merged to form a single vertex. The Petersen graph has both of these as minors, so it is by all means non-planar. Figure 6 helps to see that the Petersen graph contains K5 as a minor (by deleting the 5 edges between the pentagon and the pentagram).
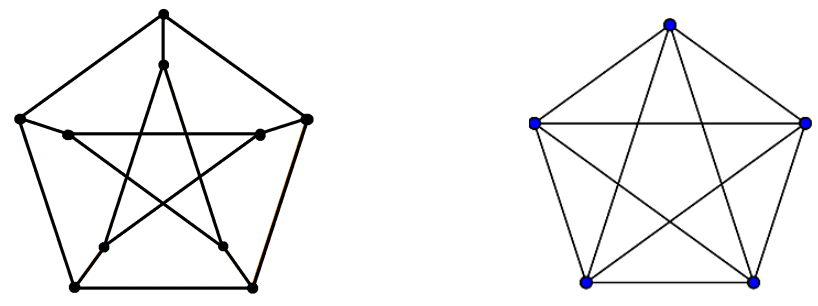

Figure 6: The Petersen and K5 graphs

\section{A unit distance graph}

The Petersen graph is a unit distance graph: it can be drawn in the plane with each edge having unit length. Figure 7 helps to see that the unit distance visualisation of the Petersen graph (on the right) can be obtained from the symmetry of order 5 drawing (on the left). This can be done by clock-wise rotation of the inner pentagram stretching the edges until they become the same length as the sides of the pentagon and the pentagram (twisting the star clock-wise). 


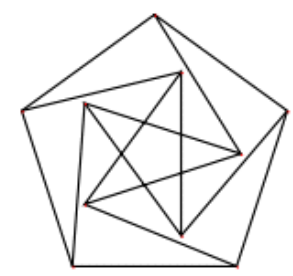

Figure 7: A unit distance drawing of the Petersen graph.

\section{A snark}

This is probably the most intriguing property of the Petersen graph, even associated with a fictional hero. First snarks were hard to find and at the same time, highly intriguing. That explains best Martin Gardner's choice for a fictional character rather than for a precise abbreviation of "Nontrivial Uncolourable Trivalent". ${ }^{12}$ The name was borrowed from Lewis Carroll's poem The Hunting of the Snark about a mysterious creature with an unusual combination of features. A snark is a graph in which every vertex has three neighbours (meaning it is a cubic graph), and the edges cannot be coloured by only three colours without two edges of the same colour meeting at a point. Again the Petersen graph stands out. Not only was it the first found snark, it is probably the smallest snark: all the other possible snarks are likely to be reducible to it (by deleting some edges and collapsing the correspondent vertices). This is a conjecture due to W. T. Tutte: every snark has the Petersen graph as a minor. ${ }^{13}$ Interestingly this conjecture is a strengthened form of the famous four colour theorem! ${ }^{14}$

\footnotetext{
${ }^{12}$ See Introduction to Gardner [1976].

${ }^{13}$ See Tutte [1966]. Thomas [1999] announced a proof of this conjecture.

${ }^{14}$ This is because (i) any graph containing the Petersen graph as a minor must be non-planar by non-planarity of the Petersen graph, and (ii) the four colour theorem is equivalent to the statement that no snark is planar (the theorem that opens the study of snarks due to Tait [1880]) or in topological terms, no snark embeds on a sphere.
} 


\section{Discussion}

\section{About the beauty of the abstract}

These are just some of the remarkable properties of the Petersen graph, the simplest to explain. ${ }^{15}$ Why should we accept that this combination of properties makes the Petersen graph beautiful? Given that most of the remarkable and rare properties of the graph are connected with its high symmetry, why would the fact that symmetries help us to grasp the mathematical structure and other properties, imply that symmetries help to make the object beautiful? Someone might wonder: do any properties of the object which help us to discover its structure contribute to its beauty? Similarly, how and why do the symmetry-related properties of the object contribute to its beauty? It may seem that we are making the aesthetic value of the object depend on the intellectual value of a certain type of property. Cain Todd [2008] raises this question in his paper challenging philosophers to differentiate epistemic and aesthetic dimensions. In fact sometimes it may be difficult to give a test to separate pleasure from epistemic or practical benefits and genuine aesthetic pleasure. ${ }^{16}$ It is often so in moral cases: something immoral we also perceive as ugly and moral as beautiful. Also there are useful and not very beautiful things and vice versa, and mathematicians seem to be aware of this fact. For example, all proofs serve the primary epistemic function of establishing their conclusion, but not all of them are judged beautiful; also, some proofs which have the additional epistemic merit of explaining their conclusions are not judged beautiful, and some which are judged beautiful do not explain their conclusions. ${ }^{17}$

\footnotetext{
${ }^{15}$ There is an entire book devoted solely to the Petersen graph, where a colossal number of remarkable properties of the graph can be found: The Petersen Graph Holton and Sheehan [1993].

${ }^{16}$ However, there are contexts when the roles seem to be more clear. For example, Cellucci [2015], p.15 suggests: "Mathematical beauty can have a role in the context of discovery, because it can guide us in selecting which hypothesis to consider and which to disregard. Therefore, the aesthetic factors can have an epistemic role qua aesthetic factors".

${ }^{17}$ See section 4 in Giaquinto [2016].
} 
One reason for accepting that these properties contribute to the beauty of the Petersen graph is that experts agree in citing them as relevant: mathematicians I have spoken with referred to these and other properties in defence of their claim that the Petersen graph is a beautiful graph. This convergence of opinion may be not decisive, because (a) it is possible that the mathematicians are using the word "beautiful" loosely and (b) even if the word is being used in a strictly correct way, experts may be wrong in identifying what is giving them aesthetic pleasure in attending to the object. While it is possible that they describe the graph as "beautiful" meaning only that it is fascinating or remarkable, we have to ask why in that case do they so often use an aesthetic expression here, when they are perfectly capable of finding non-aesthetic words to express their interest ("fascinating", "remarkable", "noteworthy", "intriguing" and alike). The most plausible explanation of their using "beautiful" is that they experience a kind and degree of pleasure that is similar to the pleasure experienced when attending to things which they feel are strictly beautiful, such as fine musical compositions or natural scenes. In this case, loose usage is not a serious possibility. While they may be wrong about exactly which combination of properties lead them to judge the Petersen graph beautiful, the properties they mention are very likely to be relevant. This is because they agree: no graph theorist (of those spoken to) thinks that one or more of the properties presented above is not a contributor to the aesthetic quality of the Petersen graph. Someone can ask: but does the beauty of the Petersen graph not depend on its having a pretty representation (unlike the Hoffman-Singleton graph which is too complicated)? I think not. Even the most beautiful drawing of the Petersen graph is not perceptually strikingly beautiful. Its visual attraction is not sufficient for explaining the strong emotional response that mathematicians have to the graph itself. Moreover, when they talk about what is beautiful about this graph, they do not mention the drawing: 
"The Petersen graph is a beautiful graph. At least, that is what graph theorists will tell you, time and again... [I]n spite of its small size - only 10 vertices and 15 edges - its structure is beautifully symmetric, and this has far reaching consequences" Erickson [2014].

"If you are talking about theoretical beauty, Petersen graph would top the list. Simple, but almost always counterexample to the simple theorems you try to cook up" Ashwin [2014].

Remarkably, one comes across with other positive emotional responses to the properties of Petersen graph which are not aesthetic, but definitely practical. Graph-theorists like testing conjectures on the graph and express their satisfaction in non-aesthetic although positively emotional terms ("falling in love with this graph", "being faithful to", "wanting to marry it" and alike). This can serve as a strong evidence for believing that at least some mathematical aesthetic judgements may be correct, as I believe the two above are. When mathematicians are judging the Petersen graph as beautiful they do not seem to be concerned with knowing and using it. These judgements are not about epistemic or pragmatic categories, but associated with mathematicians' aesthetic experience. Complex, rich and interesting symmetries unfold more structural beauty.

Zangwill has two objections to the view that mathematical entities can be beautiful. One objection relates only to entities which have an intrinsic non-aesthetic function, such as proofs. This objection is not a threat to the view that mathematical objects such as the Petersen graph can be beautiful, because mathematical graphs, like stones, can be used but have no intrinsic function. The second objection is that properties of non-sensory abstract objects simply fall outside the category of aesthetic. In support of this view Zangwill makes the following remark: "As the etymological origins of the word "aesthetic" suggest, aesthetic properties are those that we appreciate in perception." Zangwill [2001], p. 81. This implies that strictly speaking mathematicians use 
the aesthetic terms metaphorically when they have feeling about mathematical objects similar to feelings they have about art objects. "Beautiful" then, applied to mathematical entities would express a non-aesthetic value, most probably an epistemic value. As Zangwill is no doubt aware, the etymological origin of a word does not determine what the word expresses today. The fact that "ethical" originates from an ancient Greek word meaning "habit" or "custom" does not entail that only what is habitual or customary can be ethical. Similarly, the fact that "aesthetic" originates from an ancient Greek word meaning "perceive" does not entail that only what is perceivable can have aesthetic value. So the etymological consideration is not strong support for Zangwill's second objection. In defending the view that mathematical proofs can be elegant against this objection, Barker [2009] points out that one could simply stipulate that aesthetic properties and responses are sensory. (Zangwill himself does not do this.) Barker's response to such a move is appropriate here: "One could, of course, simply stipulate that aesthetic properties and responses are in some sense sensory. Nothing prevents us from using the terms "elegance," "beauty," etc., in that way if we so choose. But it is hard for me to see any real benefit in making such a stipulation. Quite the opposite: it will simply blind us to the real and important similarities that exist between mathematical and sensory beauty" Barker [2009], p. 14. Indeed, along with mathematics, most of conceptual art, where artefacts are almost irrelevant, fall out of aesthetic considerations exactly because the beauty is not necessarily perceptual. Similarly in mathematics, perceptual attractiveness is, with rare exceptions, irrelevant, and perceptual mediators require mathematical interpretation to be appreciated, as it is in the case of tangram and in the case I am going to consider next. Besides, in mathematics, it takes time to develop a good level of expertise, not to say aesthetic sensitivity. 


\section{About the beauty of visual mathematical representations}

Remember that any planar representation of the Petersen graph cannot show all its 120 symmetries. This fact clearly implies a distinction between the mathematical object and its representations. Let us now consider mathematicians' judgements about visualisations of mathematical objects, and try to understand what role perceptual properties play in such judgements. Compare the drawings of the Petersen graph in Figure 3. In the standard drawing 10 geometrical symmetries (5 rotations and 5 reflections) are easily noticed, whereas in the Kempe drawing (3b) only 6 geometrical symmetries (3 rotations and 3 reflections) are easily noticed, and the drawing $3 \mathrm{a}$ is even less revealing. The standard shows a maximum number of symmetries, is the most favourite for graph theorists, and most often found in the literature. Could it be just perceptual attractiveness of visible symmetries that makes it favourite?

To tackle the question of the relation of perceptual properties to aesthetic quality, I will consider a case when two drawings of the same graph have the same number of symmetries. Obviously by simply pulling the vertices of the pentagram outside of the pentagon one can obtain an isomorphic drawing as depicted in the right in Figure 8. (One could also have one with the pentagon within the central area of the pentagram.) This drawing is hardly ever used, it does not appear naturally in practice, and according to graph theorists, they come across with it occasionally via computer visualisations. ${ }^{18}$

Both drawings have the same five reflection and five rotation symmetries. In fact ten

is the maximum number of symmetries visualisable in a plane drawing of the graph. ${ }^{19}$ However, there is a big difference in mathematicians' aesthetic judgements of the two. Graph theorists judge the left one to be significantly more beautiful than the right one and sometimes even call them "the beautiful" and "the ugly". For a lay person

\footnotetext{
${ }^{18}$ After the lecture by Klin [2014].

${ }^{19}$ This is stated in Hong and Eades [2005]. The paper discusses mathematicians' preferences in graph drawing.
} 
they may appear equally beautiful or the right drawing may look prettier. In fact the majority prefers the right one "because it is a star". What are the experts actually responding to when saying that the left drawing is "most beautiful" and the other is "ugly", given that they have the same symmetry and represent the same mathematical structure?
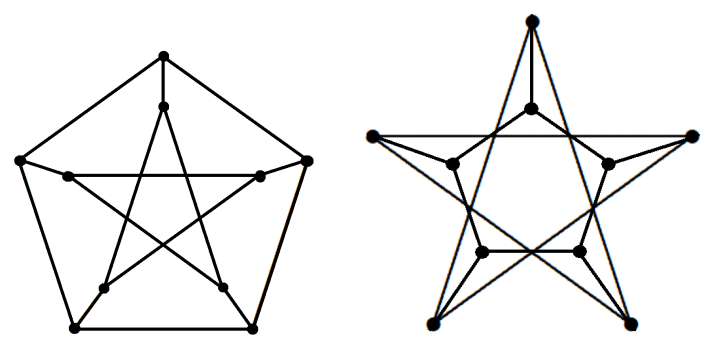

Figure 8: Two isomorphic drawings of the Petersen graph of symmetry 5.

The left drawing has more "good" properties, such as fewer edge intersections and more wide angles. ${ }^{20}$ More precisely, there are five intersection points in both drawings but in the right one three edges meet at each intersection point, instead of two. So in the right drawing at each intersection point there are three edge-intersections: if $a, b, c$, are the intersecting edges, $a$ meets $b, a$ meets $c, b$ meets $c$. Also broader inner angles mean broader space between the edges incident at a node keeping the same size of the overall drawing.

Fewer intersections with broader inner angles in the left one makes clear that it is composed of a pentagon and a pentagram (and five edges bridging them). Perceiving this geometric structure that links the graph to geometry may add to mathematical beauty of the drawing. ${ }^{21}$ This is an aesthetic merit and it adds to my emphasis on

\footnotetext{
${ }^{20}$ About efficient graph drawing see e.g. Purchase et al. [2012].

${ }^{21}$ The referee noticed that the right hand side drawing also suggests a geometric interpretation (a composition of five isosceles triangles). In fact, geometric graph theory and topological graph theory are based on geometric representations of graphs (for details see e.g. Lovász [2014] and Pisanski [2000]). I give an

example when graphs played a role of inter-mediators between group theory and hyperbolic geometry in e.g. Starikova [2012].
} 
the distinction between a representation of an object and the object itself. The two geometric subgraphs, are so well-articulated in relation one to another, that with a bit of mental transformation, one can easily turn "the beautiful" drawing into K5 and realise that K5 is a minor of the Petersen (see Figure 6). This visible similarity between the drawings of the Petersen and K5 immediately reveals that the Petersen graph is non-planar and makes it surprising that, while it is like K5 in being vertex-transitive, it is unlike $\mathrm{K} 5$ in being non-Cayley.

Moreover, observing Figure 8 of "the beautiful" and "the ugly" drawings of the Petersen graph, one can notice that "the beautiful" shows a lot of other interesting structural information, which is obscured in "the ugly". The most intriguing properties of the graph from section Rich in symmetry are visible in the traditional drawing. For example, using (a magnified) image of the visual representation of the HoffmannSingleton and the traditional visual representation of the Petersen graph in Figure 4, one can see that the Petersen graph is a subgraph of the Hofmann-Singleton graph. Furthermore, the a simple twist of the pentagram relative to the pentagon keeping the centre fixed and allowing the bridging edges to stretch shows that the graph is unit distance (see Figure 7). In the study of snarks, it is "the beautiful" drawing which "reveals" that other snarks have the Petersen graph as a minor. Notice, that the isomorphic right-hand side drawing does not provide such insights. Therefore, the abstract beauty of the graph shines the best through "the beautiful" drawing and is obscured in "the ugly". Similarly to mathematicians trying to visualise abstract structures in the most effective way, artists follow the "rules of perception" (proportions, density of details, composition, symmetry) to help a perceiver recognise beauty in the artefact.

This strengthens the case for saying that the mathematicians' aesthetic judgement in favour of the traditional drawing arises from intellectual pleasure as opposed to merely sensory pleasure. Some of the relevant mathematical properties are revealed by 
means of visual properties of the drawing. So the intellectual pleasure in this case has a sensory mediator, but mathematicians seem to respond to the intellectual content. The question is whether the judgement in favour of the traditional drawing is an aesthetic judgement, and what roles intellectual and sensory components are in this judgement. Since the function of the drawing is to help us understand the graph, this could still be a case in which the drawing performs its function more beautifully than other drawings. One can say that the value of the drawing in this case is merely instrumental, non-aesthetic, and then Zangwill's objection applies. Zangwill uses the idea of beauty independent of fulfilling a purpose from Kant, although with modification. ${ }^{22}$ However, in one passage Kant suggests that a feature which facilitates understanding may also contribute aesthetically:

"Objects that ease our perception of them give us pleasure and are beautiful... Symmetry eases our understanding and is the proportion of sensibility. Looking at a disproportional house, I find it difficult to conceive it as a whole... Uniformity of the parts helps my representation, increases my inner life, and I therefore must find it beautiful." 23

Holding a subjectivist position about mathematical beauty, Kant would not say that understanding of the representation adds to mathematical beauty of the abstract. ${ }^{24} \mathrm{He}$ said: "While mathematical properties themselves are not beautiful, it is the demonstration of such properties that can be the object of aesthetic appreciation... The purposiveness of mathematical properties does not indicate beauty but a form of perfection." 25 Wenzel in his comments on Kant's view that there is no beauty for mathematical objects

\footnotetext{
${ }^{22}$ See Zangwill [1999].

${ }^{23}$ These come fromWenzel [2001] p. 426, where he refers to (Kant 1772/73, XXV 181). According to Wenzel, Kant eventually changed his mind towards rejecting idea of mathematical aesthetics, but at least until the 1790 s Kant kept this view.

${ }^{24}$ Breitenbach sharpens this contrast of Kant's subjectivism with a Platonist's objective beauty on the p. 2.

${ }^{25}$ Borrowed from Breitenbach 2013b, p. 2, from Critique of Judgment, $\S 62$.
} 
and "mathematics by itself is nothing but rules", ${ }^{26}$ supposes that Kant underestimated the richness of mathematical practice in its historical dynamics. I believe that the case I have presented in this paper supports the view that abstract mathematical objects can be beautiful.

The interpretation in Breitenbach [2015] of Kant's aesthetic theory shades light on the Kantian rejection of beauty of mathematical abstract objects, as it is often received, and suggests that "the experience of beauty in mathematics is grounded not in an intellectual insight into particular properties of mathematical objects but in our felt awareness of the imaginative processes that lead to mathematical knowledge" (Breitenbach [2015], p.2). This implies that Kant aims to distinguish knowing the properties of the abstract object (properties that may converge to some perfection) and free (spontaneous) imaginative activities involving these properties. Only these activities, which are a subject to creativity, make possible aesthetic appreciation of such perfection. These two types of pleasure: epistemic and aesthetic, differ; and Zangwill's objection does not hold.

Kant's view is a strong subjectivist's position but it stresses an under-appreciated aspect of mathematical practice, which is still important - the practice of visualising and representing mathematical abstracta in a most comprehensible way. (Maybe more than that is also under-appreciated: responding emotionally and creatively, experimenting, going beyond standard ways in reasoning.) Clearly many mathematical areas develop their specific styles of visual representations: consider geometry, graph theory and algebra. Apparently, the art of sculpturing mathematics includes designing and refining the looks of its elements. These definitely have an aesthetic character. In practice it is often difficult to produce a mathematically effective visual representation, one which easily conveys the content. Mathematicians may react in a very favourable way to such

\footnotetext{
${ }^{26}$ See Wenzel [2001] citing Kant Refl. 922, XV 410, 1776-1778 on p. 416.
} 
exemplars. This is so with regard to the favourite drawing of the Petersen graph.

Therefore, according to Kant, visible symmetry of an object may contribute both to understanding and to beauty. Indeed the examples above demonstrate that in mathematics an aesthetic response requires at least some and sometimes very profound understanding. If that is right, the same may be true of those properties of the favourite drawing of the Petersen graph which most help one understand the graph itself. So, the favoured drawing of the Petersen graph has intellectual beauty, i.e. beauty that requires mathematical interpretation of the drawing to appreciate. But perfect and exceptional properties of the graph itself still have to be there.

To sum up this section, it is unlikely to be a mere coincidence that the "good" drawing is also considered as more beautiful. On the graph theorists' view the traditional drawing wins. The reason for that is perhaps that experts respond to the combination of the perceptual and intellectual, but presumably intellectual factors have more weight in mathematical beauty than perceptual factors. The intellectual factors are those concerning the manner of representation and the mathematical structure of the object itself. One principle that may be operating here is how easy it is for us to extract structural information directly from the drawing, or the signal-to-noise ratio. In the right hand diagram there is more irrelevant information: three times as much for each intersection point, as explained above. Moreover, the structurally significant subgraphs are not clearly separated in the right hand drawing, whereas the insignificant properties, e.g. three-edge crosses, become louder. These properties reduce the signal-to-noise ratio in the right hand drawing. Looking at a "beautiful" drawing, a mathematician more easily and more quickly discerns the structure and therefore its beauty. In contrast, when the mathematician looks at the "ugly" drawing, the extra noise diminishes her aesthetic pleasure. It is not just the number of "good" properties that makes a drawing more beautiful. The mathematician responds emotionally per- 
haps to the structural features of the graph itself revealed to us by the drawing, such as the possession of significant substructures having shapes of geometric figures. Similarly in the tanrgram example, as soon as we understand the interplay between the visualisations and mathematics behind them, we understand the visual paradoxes. Then we are able to appreciate the beauty of tangrams not simply for palette or proportions.

On the basis of this case study we can put forward the following tentative hypotheses:

- Different drawings of the same object may reveal different mathematical properties (symmetries).

- Visible symmetries may contribute to the mathematical beauty of a representation of a mathematical object (though the effect is not proportional to the number of symmetries).

- Particular visual properties help one grasp mathematical properties (structural) and appreciate it aesthetically.

- If two drawings of an object have a similar degree of perceptual beauty, the one which reveals a greater degree of mathematical beauty of the object has greater mathematical beauty.

- Mathematicians respond to a combination of various factors: perceptual and intellectual; but intellectual factors prevail.

\section{Conclusions}

Taking into account that in practice mathematicians work with visual representations, we must distinguish between beauty of mathematical objects and beauty of visual representations of mathematical objects. Visual mathematical representations may 
look aesthetically appealing both for a lay person and a mathematician. However, while a lay person appreciates solely the visual appearance of a representation, for a mathematician, a visual representation of a mathematical object may be assessed for perceptual beauty, for intellectual beauty, or a combination of both.

Visible symmetry is one mathematical property that contributes to perceptual beauty. Mathematicians find the most symmetric drawing of the Petersen graph the most beautiful. However, between two equally symmetric representations of the same Petersen graph they choose the clearer one as more beautiful. It turned out that they find visible symmetry along with other perceptual properties of graph drawings (e.g. fewer edge intersections, broader angles between adjacent vertices) also aesthetically appealing. This suggests that when comparing diagrams of the Petersen graph in aesthetic terms, mathematicians respond to a combination of both perceptually appealing properties (e.g. visible symmetries) and the mathematical properties of the object represented. A representation's possession of properties that are useful is not by itself sufficient for mathematical beauty.

Mathematicians may also make aesthetic judgements about a mathematical abstract object as such, focusing on its mathematical properties. For example, in explaining why they describe the Petersen graph as beautiful, they tend to emphasise its high symmetry relative to its small size, and its outstanding mathematical properties, mostly related to symmetry. This implies that both visible symmetry and abstract symmetry contribute to mathematical beauty.

\section{References}

Jacob Ashwin. What is the most beautiful graph you have ever seen?, 2014. URL http: //www . quora.com/Whats-the-most-beautiful-graph-you-have-ever-seen. Ac- 
cessed: 2015-09-30.

John Barker. Mathematical beauty. Sztuka i Filozofia (Art and Philosophy), Special Issue(35):60-74, 2009. doi: ISSN1230-0330.

Norman Biggs. Algebraic Graph Theory, Cambridge Mathematical Library. Cambridge University Press, 2 edition, 1994.

Angela Breitenbach. Beauty in proofs: Kant on aesthetics in mathematics. European Journal of Philosophy, 23(4):955-977, 2015.

Malcolm Budd. Aesthetic Essays. Oxford University Press, 2008.

Carlo Cellucci. Mathematical beauty, understanding, and discovery. Foundations of Science, 20(4):339-355, 2015.

Rafael De Clercq. Beauty, pages 299-308. Routledge, 3 edition, 2015.

Alejandro Erickson. Five 2-colourings of the Petersen graph, 2014. URL http:// alejandroerickson. com/home/blog/blog. php?entry=19. Accessed: 2015-09-30.

Martin Gardner. Mathematical games. Scientific American, (April):126-130, 1976.

Marcus Giaquinto. Mathematical proofs: The beautiful and the explanatory. Journal of Humanistic Mathematics, 6(1, January):52-72, 2016. doi: 10.5642/jhummath. 201601.05. URL http://scholarship.claremont.edu/jhm/vol6/iss1/5.

Alan Goldman. Aesthetic Value. Westview Press, 1995.

Adam Goucher. Ten things you (possibly) did not know about the Petersen graph, 2013. URL https://cp4space.wordpress.com/2013/09/06/ ten-things-you-possibly-didnt-know-about-the-petersen-graph/. Accessed: 2015-09-30. 
Derek Allan Holton and James Sheehan. The Petersen Graph. Cambridge University Press, 1993.

Seok-Hee Hong and Peter Eades. Drawing planar graphs symmetrically, ii: Biconnected planar graphs. Algorithmica, 42(2):159-197, 2005.

Alfred Kempe. A memoir on the theory of mathematical form. Philosophical Transactions of the Royal Society of London, 177(0):1-70, 1886. doi: 10.1098/rstl.1886.0002.

Wagner Klaus. Über eine Eigenschaft der Ebenen Komplexe. Mathematische Annalen, 114:570-590, 1937. URL http://eudml.org/doc/159935.

Mikhail Klin. Two incarnations of the Petersen graph, 2014. Computer Algebra Experimentation with Coherent Configurations, Lecture.

Mikhail Klin and Matan Zivav. Computer Algebra Investigation of Known Primitive Triangle Free Strongly Regular Graphs. URLhttp://citeseerx.ist.psu. edu/viewdoc/summary?doi=10.1.1.418.1432, 2014. Online; accessed 29 August 2016.

Jerrold Levinson. Beauty Is Not One: The Irreducible Variety of Visual Beauty. Oxford University Press, 1 edition, 2011.

László Lovász. Geometric representations of graphs. preprint on webpage at http://www.cs.elte.hu/Iovasz/bookxx/geomrep-old2.pdf, 2014.

Sam Loyd. The eighth book of Tan - 700 Tangrams by Sam Loyd with an introduction and solutions by Peter Van Note. New York: Dover Publications, 1968.

Julius Petersen. Sur le théorème de Tait. L'Intermédiaire des Mathématiciens, (5): $225-227,1898$. 
Tomaz Pisanski. Bridges between geometry and graph theory. In Catherine Gorini, editor, Geometry at Work, MAA Notes 53, pages 174-194. America, 2000.

Helen Purchase, Christopher Pilcher, and Beryl Plimmer. Graph drawing aesthetics created by users not algorithms. IEEE Transactions on Visualization and Computer Graphics, 18(1):81-92, 2012.

Jerry Slocum. The Tao of Tangram. Barnes and Noble, 2001. ISBN 978-1-4351-0156-2.

Irina Starikova. From practice to new concepts: Geometric properties of groups. Philosophia Scientiae, 16(1):129-151, 2012.

Peter Tait. Remarks on the colourings of maps. Proc. R. Soc., (10):729, 1880.

Robin Thomas. Recent excluded minor theorems for graphs, 1999. URL http:// people.math.gatech.edu/ thomas/PAP/bcc.pdf.

Cain S. Todd. Unmasking the truth beneath the beauty: Why the supposed aesthetic judgements made in science may not be aesthetic at all. International Studies in the Philosophy of Science, 22(1):61-79, 2008.

Fu Traing Wang and Chuan-Chih Hsiung. A theorem on the tangram. The American Mathematical Monthly, 49(9):596-599, 1976. doi: 10.2307/2303340.

William Thomas Tutte. On the algebraic theory of graph colorings. Journal of Combinatorial Theory, 1(1):15 - 50, 1966. ISSN 0021-9800. doi: http://dx.doi.org/10.1016/ S0021-9800(66)80004-2. URL http://www.sciencedirect.com/science/article/ pii/S0021980066800042.

Christian Wenzel. Beauty, genius, and mathematics: Why did Kant change his mind? History of Philosophy Quarterly, 18(4):415-432, 2001. 
Hermann Weyl. Symmetry. Princeton University Press, 1952.

Nick Zangwill. Feasible aesthetic formalism. NOUUS, 4(33):610-629, 1999.

Nick Zangwill. The Metaphysics of Beauty. Cornell University Press, 2001.

Nick Zangwill. Beauty. In Jerrold Levinson, editor, Oxford Companion to Aesthetics, pages 325-343. Oxford University Press, 2003. 